\title{
Distinguishing Mesoscale Polar Order (Unidirectional vs. Bidirectional) of Cellulose Microfibrils in Plant Cell Walls using Sum Frequency Generation Spectroscopy
}

Mohamadamin Makarem¹, Yoshiharu Nishiyama ${ }^{2}$, Xiaoran Xin ${ }^{3}$, Daniel Durachko ${ }^{4}$, Ying $\mathrm{Gu}^{3}$, Daniel J. Cosgrove ${ }^{4}$, and Seong H. Kim ${ }^{1 *}$

${ }^{1}$ Department of Chemical Engineering, Materials Research Institute, Pennsylvania State University, University Park, Pennsylvania 16802, USA.

${ }^{2}$ University Grenoble Alpes, CNRS, CERMAV, 38000 Grenoble, France.

${ }^{3}$ Department of Biochemistry and Molecular Biology, Pennsylvania State University, University Park, Pennsylvania 16802, USA.

${ }^{4}$ Department of Biology, Pennsylvania State University, University Park, Pennsylvania 16802, USA.

* Corresponding author: shkim@engr.psu.edu, shk10@psu.edu.

The supporting information contains eleven pages, with four figures and no tables. 


\section{Calculation of distances between crystals}

The aqueous suspension of rod-shape cellulose nanocrystals in the capillary tube forms a hexagonal array with the rod axis aligned along the long axis of the capillary tube. Then, the distance between crystals can be calculated by:

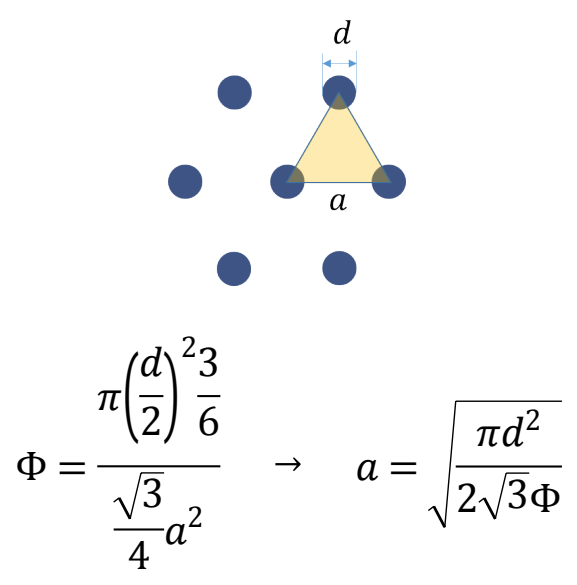

Where $\Phi$ is the volume fraction of cellulose crystal in the suspension, $d$ is the diameter of crystals, and $a$ is the distance between crystals. By considering the cross sectional diameter of the cellulose nanocrystals isolated from tunicates $(\approx 10 \mathrm{~nm})$ and the density of cellulose $(\approx 1.5$ $\mathrm{g} / \mathrm{cm}^{3}$ ), the distance between crystals is estimated to be around $58 \mathrm{~nm}$ for the cellulose content of $4 \mathrm{wt} \%$.

This model can be used for rough estimation of the average inter-CMF distance in plant cell wall. Let's assume that the elementary microfibril diameter is $\sim 4 \mathrm{~nm}$ and they are fully isolated and dispersed as in the case of liquid suspension. The typical cellulose content in the secondary cell wall is about $40 \%$ in dry mass base; then, $\Delta l$ is estimated to be about $6 \mathrm{~nm}$. In the primary cell wall, typical cellulose content is about $15-20 \%$ in dry mass base and typical water content is $70-90 \%$; then, $\Delta 1$ is estimated to be around $20-30 \mathrm{~nm}$. 

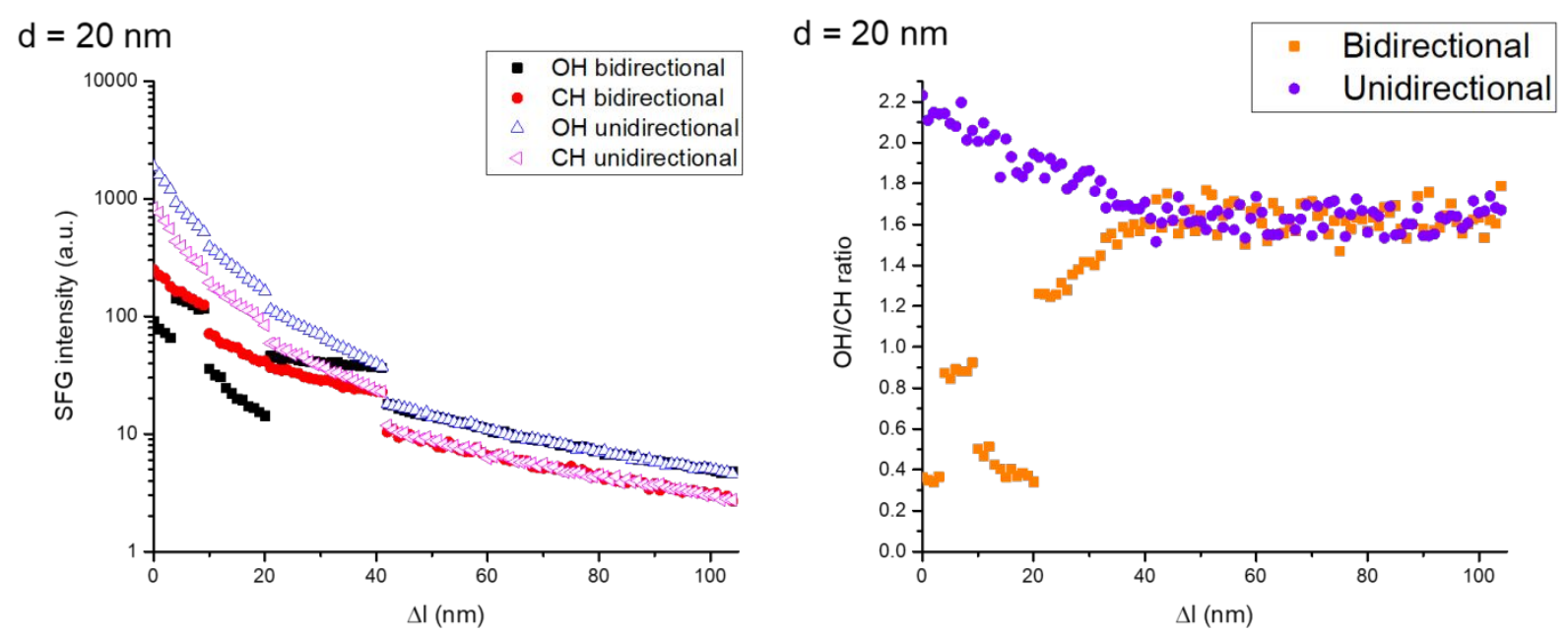

Figure S1. (a) Theoretical calculations of the $\mathrm{CH}$ and $\mathrm{OH}$ stretch peaks for $20 \mathrm{~nm}$ diameter crystals with unidirectional and bidirectional packing as a function of $\Delta l$. (b) $\mathrm{OH} / \mathrm{CH}$ intensity ratio for $20 \mathrm{~nm}$ crystals as a function of $\Delta l$ for parallel and antiparallel packing. 

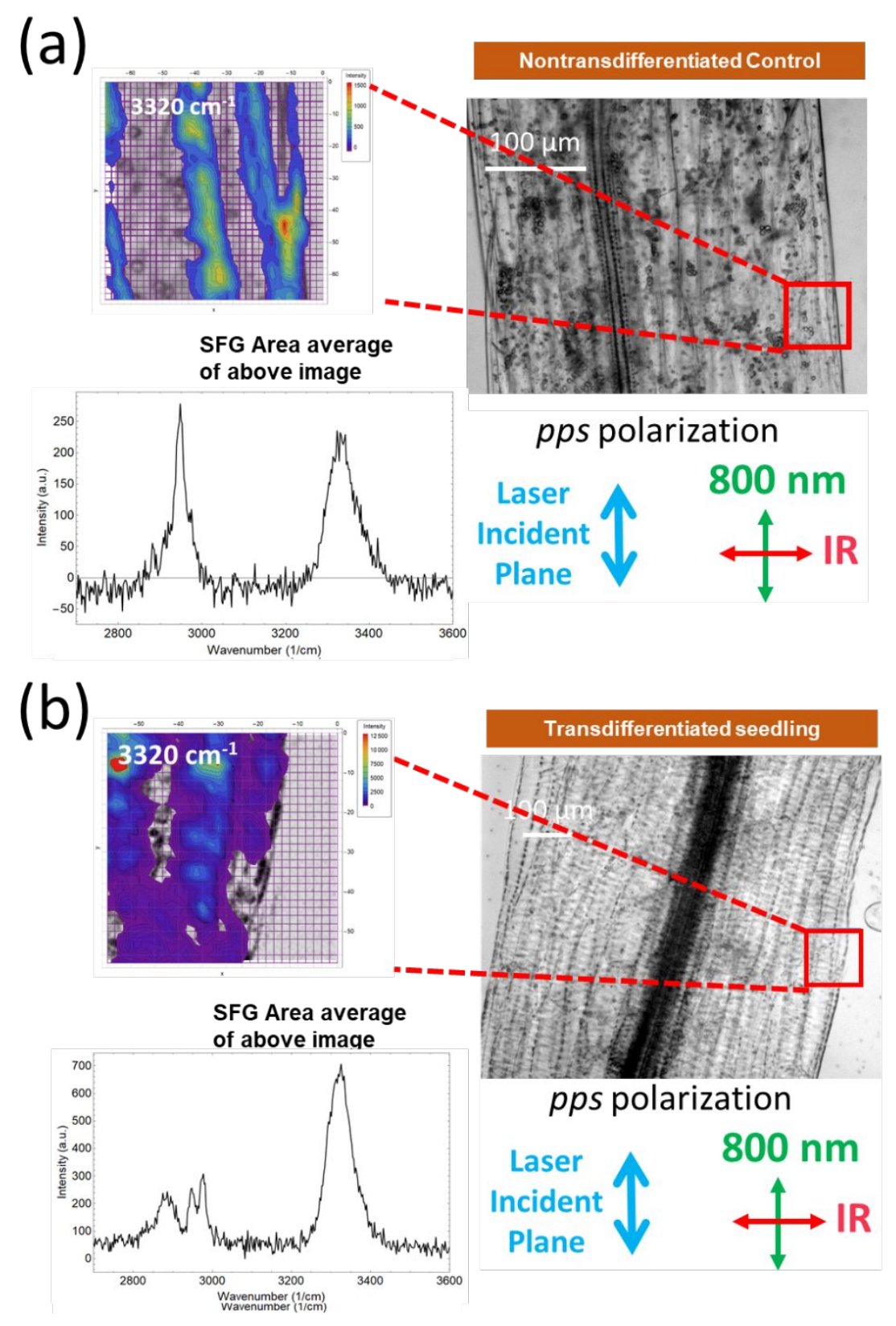

Figure S2. Control experiments of (a) nontransdifferentiated and (b) transdifferentiated epidermis of Arabidopsis hypocotyl never-dried and hydrated in $\mathrm{D}_{2} \mathrm{O}$. An area of $70 \mu \mathrm{m} \times 70 \mu \mathrm{m}$ (marked with a box) was analyzed with a $2 \mu \mathrm{m}$ step interval. The hyperspectral SFG imaging was done with the $p p s$ polarization. The nontransdifferentiated epidermis is a primary cell with low cellulose content and high water content; thus, the average inter-CMF distance is large and the $\mathrm{OH} / \mathrm{CH}$ intensity ratio is $0.7 \sim 0.8$. The xylem induced in the epidermis is a secondary cell wall with high cellulose content and low water content; thus, the average inter-CMF distance is small. Then, the $\mathrm{OH} / \mathrm{CH}$ intensity ratio of $\sim 3$ must be due to the biased unidirectionality of CMFs within the probe volume. 
(a)

(b)

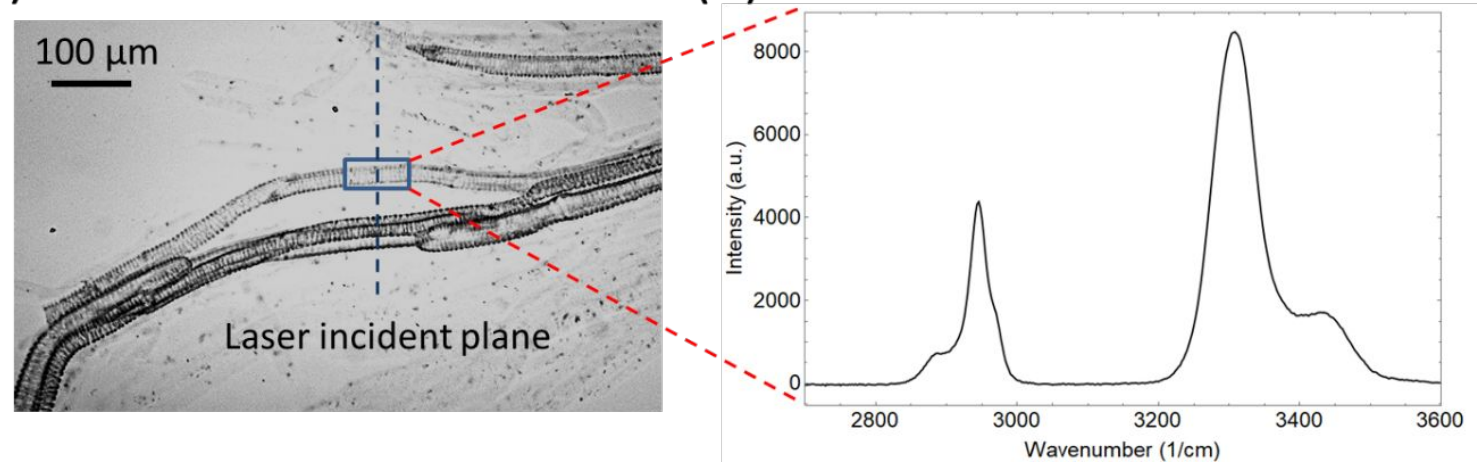

Figure S3. (a) The $90^{\circ}$ rotation of celery coils with respect to laser incident plane. (b) The average pps$\mathrm{SFG}$ spectra of the marked area. The $\mathrm{OH} / \mathrm{CH}$ ratio follows similar trend observed in Figure 7. The difference in the $\mathrm{CH}$ and $\mathrm{OH}$ peak shape is due to the azimuth orientation dependence.

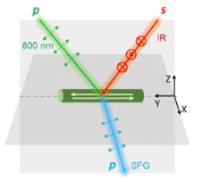

Transmission - in-plane

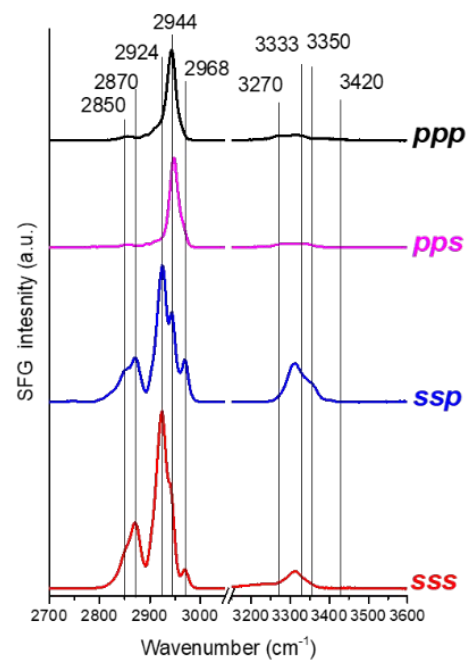

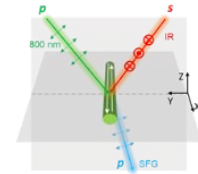

Transmission - perpendicular

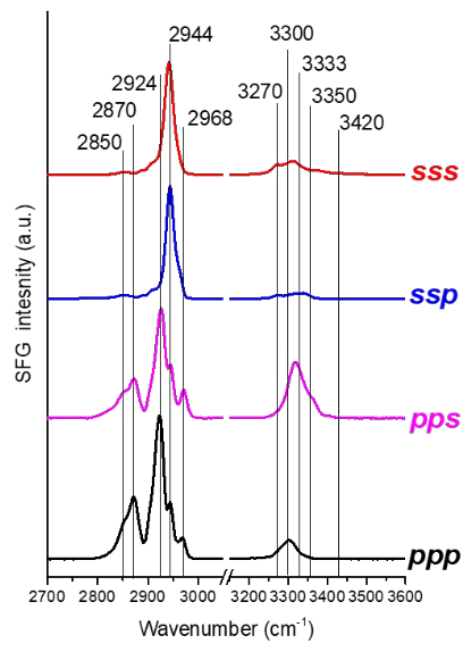

Figure S4. SFG spectra of the uniaxially-packed cellulose I $\beta$ with unbiased bidirectionality. This is the case where $\Delta \mathrm{l} \approx 0 \mathrm{~nm}$ (volume fraction $\approx 100 \%$, except some voids between crystals). 


\section{Mathematica code}

\section{(*Coherence Length Calculations*)}

$\mathrm{c}=299792458 ;\left(*\right.$ speed of light $\mathrm{m} / \mathrm{s}^{*}$ )

lambda1 $=3500 * 10^{\wedge}-9$; (*wavelength*)

lambda2 $=800 * 10^{\wedge}-9$; (*wavelength*)

lambda3 $=($ lambda $1 * 1$ ambda2 $) /($ lambda $1+1$ ambda2 $) / / N$;

6.511627906976744 *^-7;

teta $1_{\text {air }}=45 ;(*$ degrees $*)$

teta $2_{\text {air }}=45 ;(*$ degrees $*)$

(*snells law:Subscript[n, air] sin(Subscript[teta, air])=Subscript [n, cellulose] sin (Subscript [teta, cellulose])*)

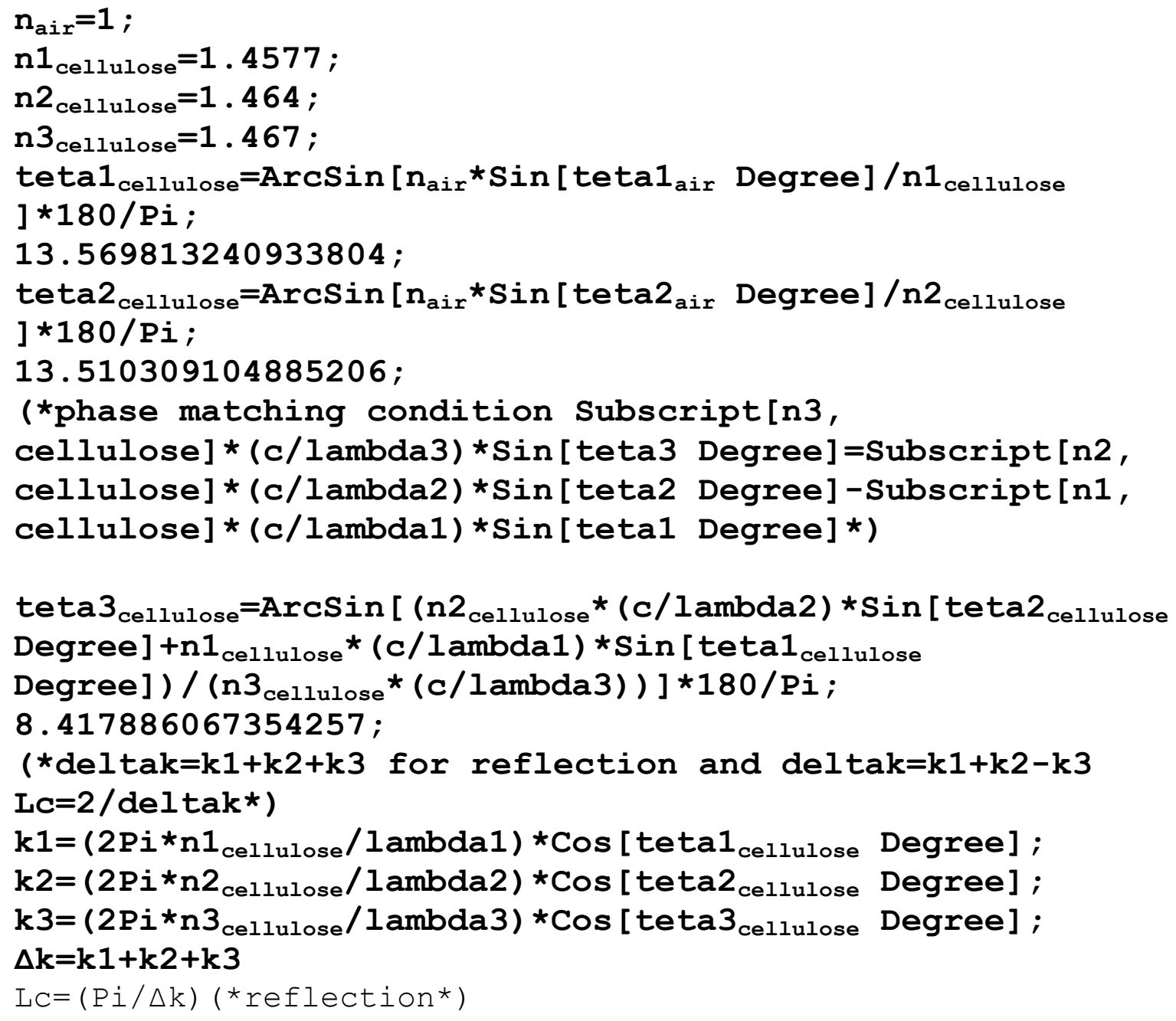


(*OH - Antiarallel*)

$\mathrm{F}=$ Table $[0,\{\mathrm{w}, 1,100\}]$;

$c=$ Table $[\{0,0,0\},\{w, 38 * 123\}]$;

$\mathrm{p}=1$;

$\mathrm{q}=1$

For $[u=3, u<41, u++$,

$$
\begin{aligned}
& \text { For }[\Delta z=0, \Delta z<123, \Delta z++, \\
& \text { c }[[(p-1) 123+q, 2]]=u ; \\
& c[[(p-1) 123+q, 1]]=\Delta z ; \\
& m=(L c * 109) /(u+\Delta z) ; \\
& \operatorname{If}[(\Delta z+u)<(L c * 109+1), \\
& \text { For }[w=1, w<101, w++,
\end{aligned}
$$$$
\text { temp }=((\operatorname{Exp}[-I \Delta \mathrm{k} u * 10-9]-1) / \Delta \mathrm{k})(\operatorname{Sum}[\mathrm{m} \text { u2 (- }
$$

1) $i * \operatorname{Cos}[$ RandomVariate [NormalDistribution $[\mathrm{Pi} / 6,0.15]]] \operatorname{Exp}[-$

I $\Delta \mathrm{k} i(u * 10-9+\Delta z \star 10-9)] \operatorname{Exp}[-i(u * 10-9+\Delta z \star 10-9)],\{i, 0, m-$ 1\}] +Sum [m u2

RandomReal [ $\{0,1\}]$ Sin [RandomVariate [NormalDistribution [Pi/6, $0.15]]] \operatorname{Exp}[-$ I $\Delta \mathrm{k}$ i $(u * 10-9+\Delta z * 10-9)] \operatorname{Exp}[-i(u * 10-9+\Delta z * 10-$ 9) $],\{i, 0, \mathrm{~m}-1\}])$;

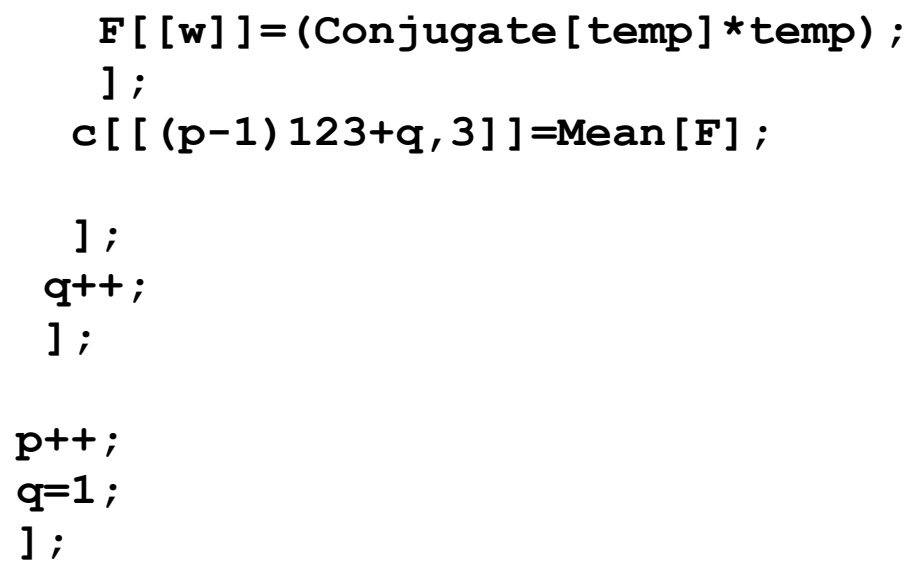


(*OH - Parallel*)

$\mathrm{G}=$ Table $[0,\{\mathrm{w}, 1,100\}]$;

$\mathrm{dd}=$ Table $[\{0,0,0\},\{w, 38 * 123\}]$;

$\mathrm{p}=1$;

$\mathrm{q}=1$;

For $[u=3, u<41, u++$,

$\operatorname{For}[\Delta z=0, \Delta z<123, \Delta z++$,

$\operatorname{dd}[[(p-1) 123+q, 2]]=u$;

$\operatorname{dd}[[(p-1) 123+q, 1]]=\Delta z$;

$\mathrm{m}=\mathrm{Lc} /(\mathrm{u} * 10-9+\Delta \mathrm{z} * 10-9)$;

If $[(\Delta z+u)<(L c * 109+1)$,

For $[\mathrm{w}=1, \mathrm{w}<101, \mathrm{w}++$, temp $=((\operatorname{Exp}[-I \Delta \mathrm{k} u * 10-9]-1) / \Delta \mathrm{k})(\operatorname{Sum}[\mathrm{m}$ $\mathrm{u} 2$ *Cos [RandomVariate [NormalDistribution [Pi/6, 0.15] ] ] Exp [- I $\Delta \mathrm{k} i(u * 10-9+\Delta z * 10-9)] \operatorname{Exp}[-i(u * 10-9+\Delta z * 10-9)],\{i, 0, m-$ 1\}] +Sum [m u2

RandomReal [ $\{0,1\}]$ Sin [RandomVariate [NormalDistribution [Pi/6, $0.15]]] \operatorname{Exp}[-$ I $\Delta \mathbf{k} i(u * 10-9+\Delta z * 10-9)] \operatorname{Exp}[-i(u * 10-9+\Delta z * 10-$ 9) $],\{i, 0, m-1\}])$;

$G[[w]]=($ Conjugate $[$ temp $]$ *temp $)$;

] ;

$\operatorname{dd}[[(p-1) 123+q, 3]]=$ Mean $[G]$;

$$
\begin{aligned}
& \text { ] } \\
& \mathrm{q}^{++} \\
& \text {] }
\end{aligned}
$$

$\mathrm{p}++$;

$q=1 ;$

]; 


\section{(*CH - Antiparallel*)}

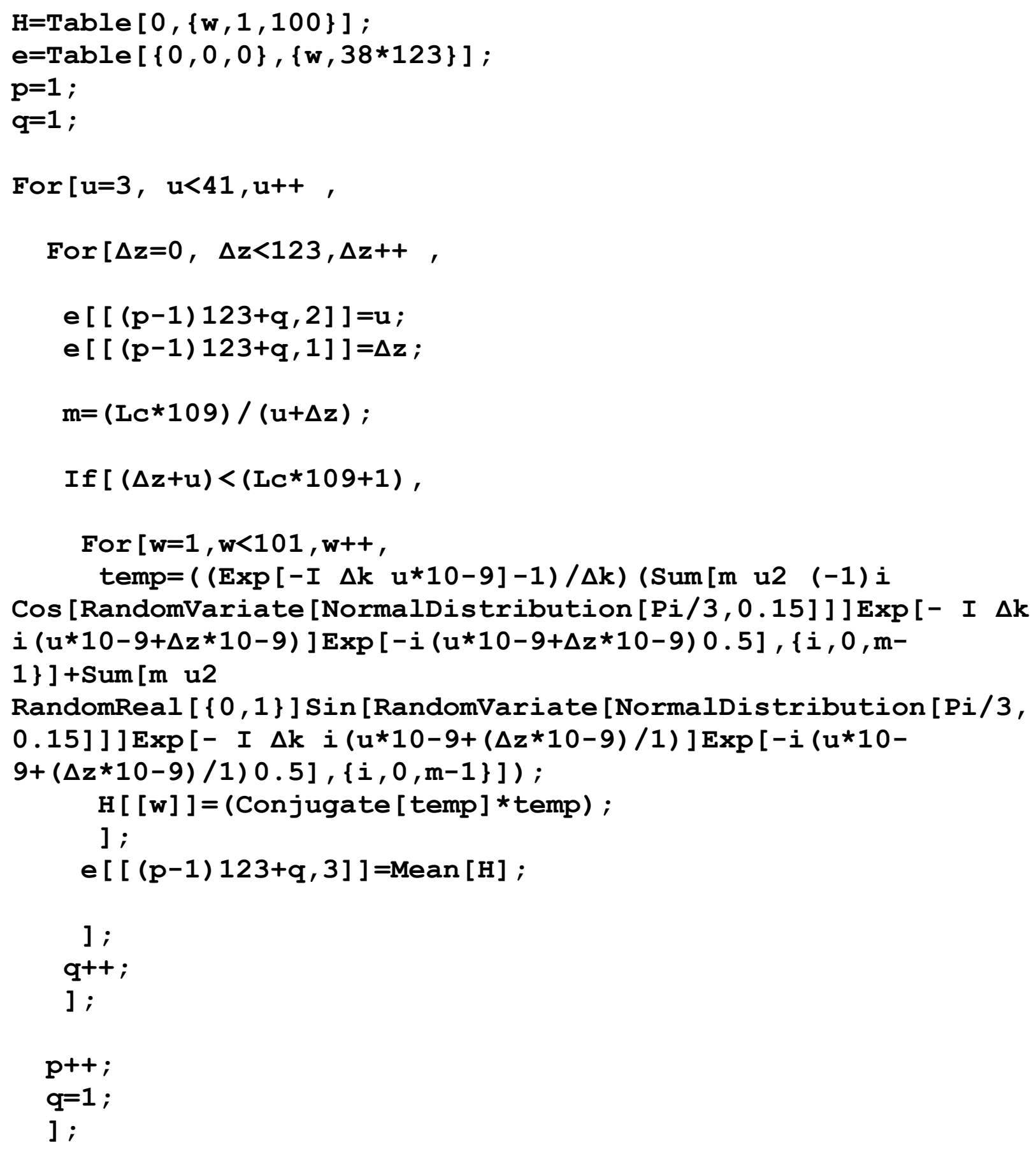


(*CH - Parallel*)

$\mathrm{J}=$ Table $[0,\{\mathrm{w}, 1,100\}]$;

$f f=T a b l e ~[\{0,0,0\},\{w, 38 * 123\}]$;

$\mathrm{p}=1$;

$q=1 ;$

For $[u=3, u<41, u++$,

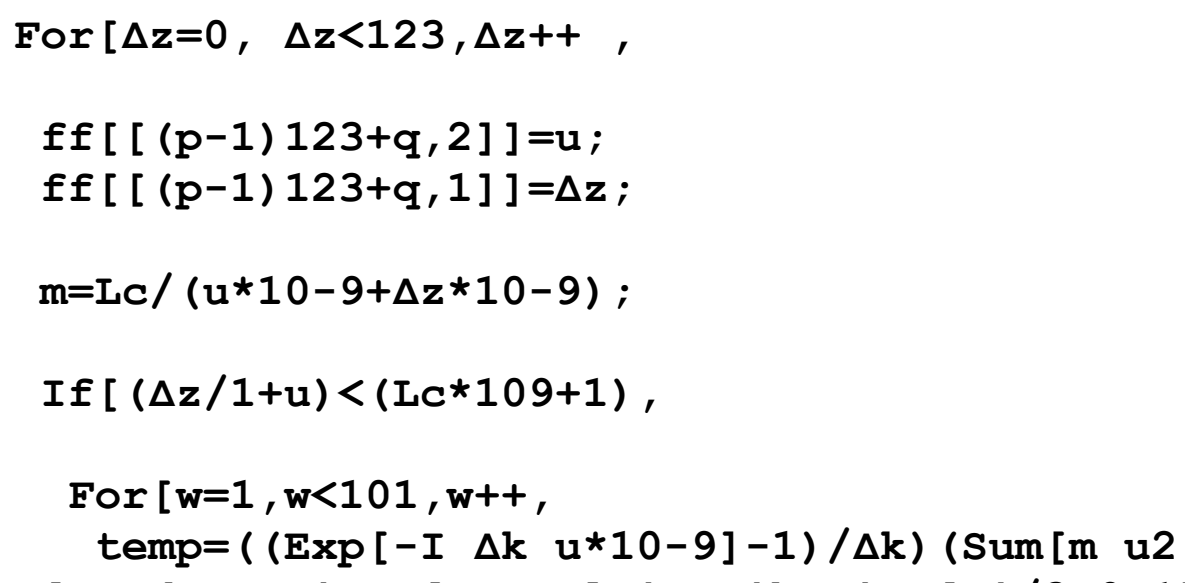

Cos [RandomVariate [NormalDistribution [Pi/3,0.15]] ] Exp [- I $\Delta \mathbf{k}$ $i(u * 10-9+\Delta z * 10-9)] \operatorname{Exp}[-i(u * 10-9+\Delta z \star 10-9)],\{i, 0, m-1\}]+\operatorname{Sum}[m$ u2

RandomReal [ $\{0,1\}]$ Sin [RandomVariate [NormalDistribution [Pi/3, $0.15]]] \operatorname{Exp}[-$ I $\Delta \mathbf{k} i(u * 10-9+\Delta z * 10-9)] \operatorname{Exp}[-i(u * 10-9+\Delta z * 10-$ 9) $],\{i, 0, \mathrm{~m}-1\}])$;

$\mathrm{J}[\mathrm{w}]]=($ Conjugate $[$ temp] * temp) ;

] ;

$\operatorname{ff}[[(p-1) 123+q, 3]]=\operatorname{Mean}[J]$;

]
q++
]

$\mathrm{p}++$;

$q=1$;

] ; 
$\mathrm{U}=5$;

$\mathrm{V}=10$;

$\mathrm{W}=20$;

$\mathrm{Y}=30$;

$\mathrm{dU}=\mathrm{Table}[\{\operatorname{Re}[\mathrm{c}[\mathrm{s}+(\mathrm{V}-1) 123,1]]], \operatorname{Re}[\mathrm{c}[\mathrm{s}+(\mathrm{U}-$

1) 123,3$]]] / \operatorname{Re}[e[[s+(U-1) 123,3]]]\},\{s, 1,123\}]$;

$\mathrm{dV}=$ Table $[\{\operatorname{Re}[\mathrm{dd}[[\mathrm{s}+(\mathrm{V}-1) 123,1]]], \operatorname{Re}[\mathrm{dd}[[\mathrm{s}+(\mathrm{U}-$

1) 123,3$]]] / \operatorname{Re}[f f[[s+(U-1) 123,3]]]\},\{s, 1,123\}]$;

$\mathrm{dX}=\mathrm{Tab} l e[\{\operatorname{Re}[\mathrm{c}[\mathrm{s}+(\mathrm{V}-1) 123,1]]], \operatorname{Re}[\mathrm{c}[\mathrm{s}+(\mathrm{U}-$

1) 123,3$]]]\},\{s, 1,123\}]$;

$\mathrm{dW}=\mathrm{Tab} l e[\{\operatorname{Re}[\mathrm{c}[\mathrm{s}+(\mathrm{W}-1) 123,1]]], \operatorname{Re}[\mathrm{e}[\mathrm{s}+(\mathrm{U}-$

1) 123,3$]]]\},\{s, 1,123\}]$;

$\mathrm{dT}=\mathrm{Table}[\{\operatorname{Re}[\mathrm{c}[\mathrm{s}+(\mathrm{W}-1) 123,1]]], \operatorname{Re}[\mathrm{dd}[\mathrm{s}+(\mathrm{U}-$ 1) 123,3$]]]\},\{s, 1,123\}]$;

$\mathrm{dH}=$ Table $[\{\operatorname{Re}[\mathrm{c}[\mathrm{s}+(\mathrm{W}-1) 123,1]]], \operatorname{Re}[\mathrm{ff}[\mathrm{s}+(\mathrm{U}-$ 1) 123,3$]]]\},\{s, 1,123\}]$;

ListPlot [ $\{$ MovingAverage [dV, 1], MovingAverage [dU, 1] \}, PlotRang e->All]

ListPlot [ [MovingAverage [dX,1], MovingAverage [dW, 1], MovingAve rage $[\mathrm{dT}, 1]$, MovingAverage $[\mathrm{dH}, 1]\}$, PlotStyle-

$>\{\{$ Black $\},\{$ Red $\},\{$ Blue $\},\{$ Green $\},\{$ Pink $\}\}$, PlotRange- $>\{A l 1$, $\left.\left.\left\{0,10^{\wedge}-10\right\}\right\}\right]$ 